\title{
Laminated Micro-Machine: Design and Fabrication of a Flexure-based Delta Robot
}

\author{
Jorge E. Correa, Joseph Toombs, Nicholas Toombs and Placid M. Ferreira \\ Department of Mechanical Science and Engineering \\ University of Illinois at Urbana-Champaign. Urbana, IL, USA \\ jcorre20@illinois.edu,pferreir@illinois.edu
}

\begin{abstract}
Mesoscale electromechanical systems find applications in fields such as medical instrumentation, soft robotics, microscopy, flexible electronics and imagining. This paper implements the printed circuit MEMS (PC-MEMS) process[1]for the fabrication of a 'pop-up' flexure-based mesoscale systemthat exploits the simplicity of 2-D manufacturing techniques such assheet-metal operations and laser cutting to realize a 3-D mechanism. The fabrication of a laminated Delta robot with prismatic actuation is presented to exemplify this process. A working device with actuation and functional components such as linear guides, stepper motors and limit switches is designed and fully realized. Because the mechanism is popped out of the plane to achieve its3D shape, we present a stiffness analysis to arrive at the out-of-plane (or 'pop-out') angles that the planar system must accommodate so that constraints/limits on actuator torque/force can be can satisfied while producing an operational system. The simplicity of the processes makes it a candidate for the use in the emerging open-source hardware technologies forfabricating low-cost, complex, electromechanical systems.
\end{abstract}

Keywords:Laminated manufacturing, Delta robot, PC- MEMS

\section{Introduction}

(C) 2016. This manuscript version is made available under the Elsevier user license

http://www.elsevier.com/open-access/userlicense/1.0/ 


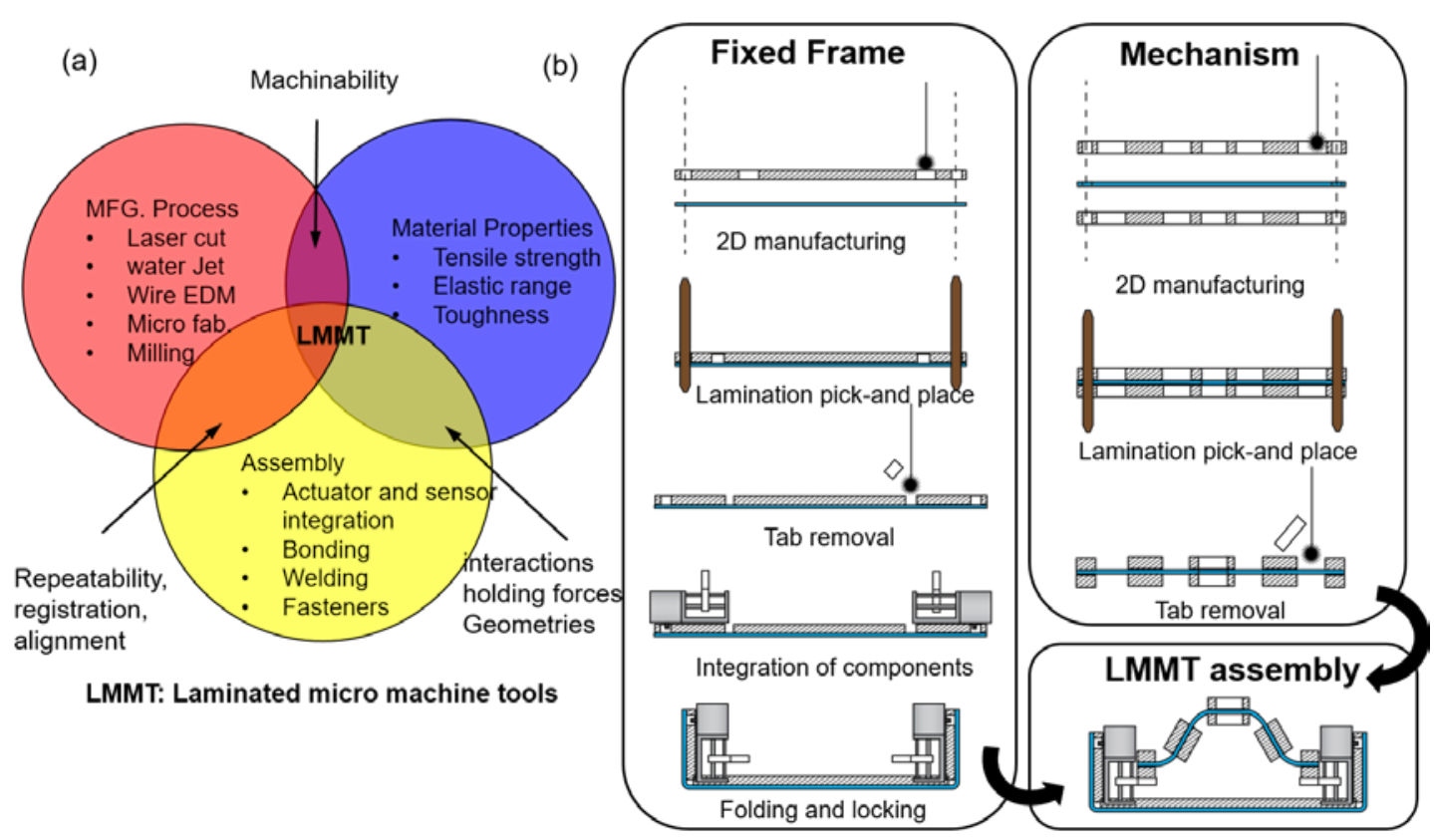

Figure 1. (a) Main technical aspects of laminated micro-machine tools (LMMT) and (b) The PC-MEMS process adapted to the fabrication of LMMT

Sophisticated industrial manufacturing techniques such as CNC milling, wire EDM, precision casting/molding and lithography-based fabricationare typically used for manufacturing micro-and mesoscale flexure-based electro-mechanical systems. These techniques have the advantages of producing robust machines with long life spans for applications ranging fromminiature robots and micro and nanopositioning systems to force/displacement sensors andoptical scanners and aligners[2]. The disadvantage ofusing these manufacturing technologies include high costs accruing from sophisticated tooling and highly-skilled labor, as well as long process and set up times. Additionally, the long set up and process times lead to slow design iterations and long development lead times.To alleviate these difficulties while achievingdesired device performance and yields, 2D manufacturing techniques such as laser cutting, water jet cutting, 2-D sheet-metal working (i.e., blanking and punching) can be used to construct multilayeredcomposites. The layer materials can be judiciously to match the function attributed to that layer (e.g., layers producing out-of-plane compliance can be made of made of spring steel) and sandwiched/laminated and bonded (resistance welded or riveted) to other layers. This strategy allows compliance to be localized and distributed to support a desired geometric design.

Emerging open source (software and hardware) platforms provide a rich offering of manufacturing technologies such as laser cutting, 3D printing[3]and CNC machining, which, in turn, open up the option of economical manufacturing of one-off complex geometries or simple shapes from a variety of polymersand affordable metals, albeit at reduced accuracies and strengths. While such approaches have the potential for use in the fabrication of mesoscale electro-mechanical systems, they required the implementation of smart and appropriatedesignrules([4],[5],[6],[7]) to alleviate the effects of low accuracy, poor surface finishing, anisotropy and reductions inmaterial properties of components, thermal instability andsmall holding forces[3].There is a strong motivation for low-cost, scalable manufacturing techniques that address the design and construction of mesoscale systems for single-use/reduced-life applications, often encountered medical industry (because of biocontamination), soft robotics, flexible electronics, and general support of machine design iterationthrough rapid functional prototyping [8]. PCB-inspired lamination techniques([9],[10],[11]) 
based on folding of multilayered rigid-flexible laminates, has been successfully shown in the fabrication of mesoscale micro-electro-mechanical systems (MEMS) with the incorporation of microscale mechanical features as piezo-actuators, integrated circuits and the integration of multiple materials (usually bulk machined with laser). Some of the examples in the literature of this technology include the Harvard Monolithic bee [1]and the hexapod crawling robot from Berkley[12].

This work presents a fabrication methodology and a stiffness-based design approach to laminated micro-machine tools (LMMT) that combines the PC-MEMS process, with conventional 2D highthroughput manufacturing techniques. In this approach, the machine frames and the mechanism structures are, first, laminated and folded individually and, later, assembled into the final devices. A Delta parallel-kinematics system[13]with three independent kinematic chains and XYZ motionis used as example to show the methodology capabilities. The machine structure is a multilayer device fabricated with sheets of polyethylene and Delrin ${ }^{\circledR}$. Due to the size of the structure and itspreference for out-out-plane motion, the pop-up folding effect is achieved without the use of scaffolds. Additionally, this paper presentsassembly and manufacturing concepts used to incorporate linear guides, limit switches, connectors and miniature stepper motors resulting in the realization and of ultra-low cost, fully-integrated, 3-Dlamination-based micromachine.

\section{Manufacturing Process Overview}

In the PC-MEMS (See Fig 1.b), micromachined layers are sequentially laminated and then micromachinedagain in an iterative process, to release the internal features and form the laminates. For instance, Figures2(a) - (c)show the basic lamination and folding steps of the Delta parallel-kinematics system.Even though the moving table and the three kinematic chains are produced in a single laminate, a single leg is shown for simplicity.In this examplethe out-of-plain hinges of are realized by clamping a thin, flexible layer between two rigid layers to both ends. The flexible layer is locally cut in the form of beams that deflect along the desire directionwhile the rigid layers offer an effective change in the section to localize the deflections in the beam. Sacrificial tabs are initially included in
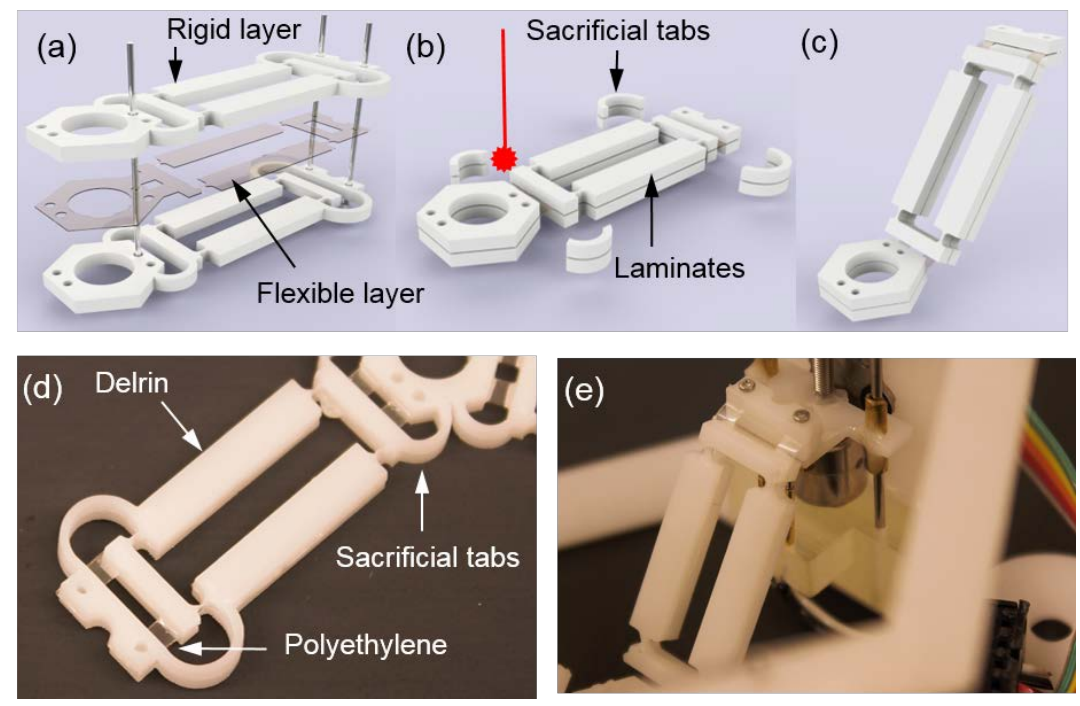

Figure 2. Lamination and folding steps of the Delta Parallel kinematics LMMT. A 3D render shows the basic steps of the process consisting of: (a) lamination of rigid and flexible layers before lamination. (b) micromachining step to release the out-of-plain joints of the robot and (c) folding the laminates. (d) and (e) show the actual laminates before and after folding. 
the fabrication of the rigid layers to keep the clamping ends connected. The tabs areremoved, in a post-micromachining step, after the flexible and the rigid layers have been laminated together. This prevent the rigid layers form been disconnected at any step of the process and, otherwise, fall apart during fabrication. The fabrication of the in-plain circular hinges is achieved by micromachining circularnotches in both the rigid and flexible layers.

The final structures are obtained by folding and locking the laminates in place. We use a 60W, $\mathrm{CO}_{2}$ laser to micromachine the individual layers with in-plane features as small as $150 \mu \mathrm{m}$. Because of the process capabilities and requirements, the Delta robot uses a polyethylene layer with a thickness of $100 \mu \mathrm{m}$ for the out-of-plain hinges, sandwiched between two $1.2 \mathrm{~mm}$ thick layers of Delrin ${ }^{\circledR}$ with the in-plain hinges (See Figure 2 (d) and (d)). Delrin ${ }^{\circledR}$ was used for because of its high stiffness and strength while polyethylene was used for its flexibility and easiness of machinability. Because of itslow surface energy, Delrin ${ }^{\circledR}$ is prepared before lamination by sanding its surface (to introduce some roughness) and using a corona plasma spot generation to change its surface chemistry. The bonding between layers is done by degreasing the surfaces and applying an adhesive epoxy. Finally, a custommade jig with alignment post (See Figure 2 (a)) is used to provide overlay registration of features during the lamination process. The resulting laminates (the frame and the mechanism structure) areintegrated with discrete components, and assembled together into the final machine as described in Section 4.

\section{Design}

The design and manufacturing process starts by identifying the requirements of the LMMT, i.e., its holding forces, overall sizes and ranges of motion. These constraints, narrow the set of candidate manufacturing processes, materials and lamination techniques (Figure 1 (a)). The Delta robot in this paper is designed to have a cubical workspace of side $4 \mathrm{~mm}$, a footprint of radius $R=50 \mathrm{~mm}$ and around $1 \mathrm{~N}$ of holding force in the $\mathrm{X}, \mathrm{Y}$ and $\mathrm{Z}$ directions. Additionally, a target of 100 microns was set for its positioning accuracy.

As shown in Figure 3.b. The laminated Delta robot is composed of identical $\mathbf{P U U}$ (prismatic actuated-universal-universal)kinematic chains, in parallel, between a fixed and the moving plate. The actuated distance of each chain is $\lambda_{i}(i=1$ to 3$)$. The universal joints in the legs are realized by parallelogram four-bar mechanisms connecting the moving plate to the controlled prismatic joint by in-plane and out-of-plane hinges. The parallelogram ensures the translational motions of the stage. 
Inthis section, the kinematic analysis of this mechanism [14]is used to derive an expression for the stiffness of the structureat the nominal configuration (pop-up angle). The length $l$ of the four-bar parallelogram and the initial pop-up angle $\alpha_{0}$ are obtained by matching the stiffness of the structure to the force-stroke capabilities of the actuators.

The Lagrange equation (derived from the Hamiltonian principle) is stated as:

$$
\frac{d}{d t}\left(\frac{\partial T}{\partial \dot{x}_{i}}\right)-\frac{\partial T}{\partial x_{i}}+\frac{\partial V}{\partial x_{i}}=Q_{i}, i=1,2,3
$$

Where $T$ is the kinetic energy of the system, $V$ is the strain energy stored in the springs and $x_{i}$ are the generalized coordinates ( $x, y$ and $z$ ). $Q_{i}$ represents the generalized forces (the external forces at the endeffector of the mechanism). In this analysis, the mass of the structure is lumped at the moving table and the springs are assumed to be perfectly elastic. If all velocities $\dot{x}_{i}=0$, the equations of motion become:

$$
\frac{\partial V}{\partial x_{i}}=Q_{i}, i=1,2,3
$$

Each of the kinematic chains has four in-plain hinges and four out-of-plain hinges, thus, the total strain energy stored in the flexible elements can be obtained as:

$$
V=4\left\{\frac{1}{2} K_{\alpha}\left[\begin{array}{lll}
\Delta \alpha_{1} & \Delta \alpha_{2} & \Delta \alpha_{3}
\end{array}\right] \cdot\left[\begin{array}{c}
\Delta \alpha_{1} \\
\Delta \alpha_{2} \\
\Delta \alpha_{3}
\end{array}\right]\right\}+4\left\{\frac{1}{2} K_{\beta}\left[\begin{array}{lll}
\Delta \beta_{1} & \Delta \beta_{2} & \Delta \beta_{3}
\end{array}\right] \cdot\left[\begin{array}{c}
\Delta \beta_{1} \\
\Delta \beta_{2} \\
\Delta \beta_{3}
\end{array}\right]\right\}
$$

Where $K_{\alpha}$ is the stiffness of the out-of-plane notch flexures and $K_{\beta}$ is the corresponding stiffness of the in-plane small flexural pivots[15][16]. $\Delta \beta_{i}$ and $\Delta \alpha_{i}$ are the in-plane and out-of-plain angular displacements of the four-bar parallelograms of the $i$-th chain (See Fig. 3.(b). For simplicity, only the in-plain and out-of-plain angular displacements of the first kinematic chain are shown).The energy stored in all springs relates to the displacement of the end-effector through the Jacobian matrices of the manipulator given as:

$$
V=2 K_{\alpha} \Delta \boldsymbol{\alpha}^{\mathrm{T}} \cdot\left[J_{\alpha}{ }^{-\mathrm{T}} J_{\alpha}{ }^{-1}\right] \cdot \Delta \boldsymbol{\alpha}+2 K_{\beta} \Delta \boldsymbol{\beta}^{\mathrm{T}} \cdot\left[J_{\beta}{ }^{-\mathrm{T}} J_{\beta}{ }^{-1}\right] \cdot \Delta \boldsymbol{\beta}
$$

Where $J_{\alpha}$ and $J_{\beta}$ are the Jacobian matrices from the linearized kinematics [17], [14] of the stage relating the displacements of the joints $\Delta \boldsymbol{\alpha}=\left[\Delta \alpha_{1}, \Delta \alpha_{2}, \Delta \alpha_{3}\right]^{\mathrm{T}}$ andjoints $\Delta \boldsymbol{\beta}=\left[\Delta \beta_{1}, \Delta \beta_{2}, \Delta \beta_{3}\right]^{\mathrm{T}}$ to the displacements of the generalized coordinates $\Delta \mathbf{x}=[\Delta x, \Delta y, \Delta z]^{\mathrm{T}}$. Substituting Eq. (1.4) into the
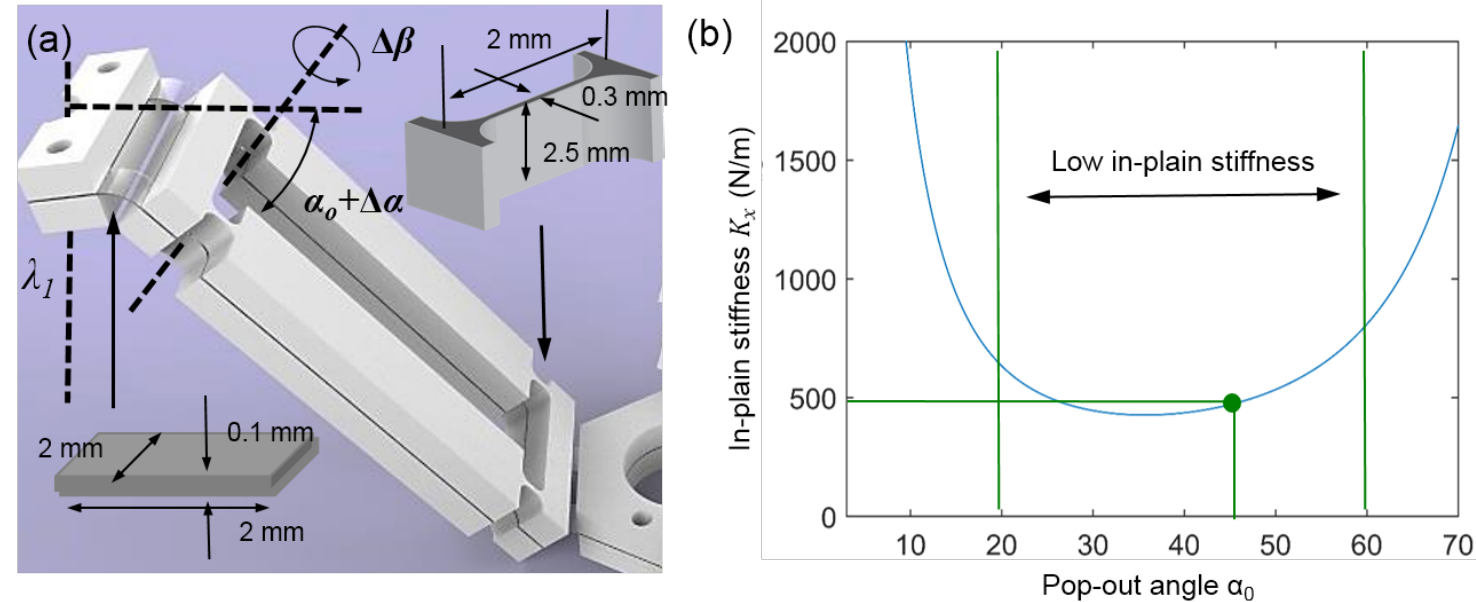

Figure. 4. (a) Dimension of the flexural hinges and (b) lateral stiffness of the mechanism as a function of the initial pop-up angle 
Lagrange's Eq. (1.2) yields the force balance equation of the machine:

$$
K\left[\begin{array}{c}
\Delta x \\
\Delta y \\
\Delta z
\end{array}\right]=\left[\begin{array}{l}
F_{x} \\
F_{y} \\
F_{z}
\end{array}\right], K=4\left[\sum_{i=1}^{3} K_{\alpha} J_{\alpha}{ }^{-\mathrm{T}} J_{\alpha}{ }^{-1}+K_{\beta} J_{\beta}{ }^{-\mathrm{T}} J_{\beta}{ }^{-1}\right]
$$

Because of the planar symmetry of the machine, $K$ is a diagonal matrix with components $K_{x}$, $K_{y}$ and $K_{z}$. Further, the instantaneous stiffness in the $\mathrm{X}$ and $\mathrm{Y}$ directions are the same while the stiffness in the Z-direction is zero. This is because of the fact that, initially, any motion along the Zdirection isboth, produced and constrained by the actuators and the structure without deflections in the compliant elements. The linearized stiffness in the plain is given by:

$$
K_{x}=K_{y}=\frac{6\left(K_{\alpha} \csc \left[\alpha_{0}\right]^{2}+K_{\beta} \sec \left[\alpha_{0}\right]^{2}\right)}{l^{2}}
$$

From Eq. (1.6), longer parallelograms result in reduced stiffness. Because of this andthe footprint design-constraint, the length $l$ is chosen to be $28 \mathrm{~mm}$. Also, based on constraints of the lasermachining process, the joints dimensions (SeeFigure (4.(a)) chosen result in torsional stiffness of $K_{\alpha}=0.0067 \mathrm{Nm} / \mathrm{rad}$ and $K_{\beta}=0.025 \mathrm{Nm} / \mathrm{rad}$. Figure 4.(b) shows the value of the in-plain stiffness after the machine has been pop-up as a function of $\alpha_{0}$. The variation of stiffness for angles between 20 and 60 degrees is relatively small. It can be shown, using force-position contragradience, that these values correspond to actuation stiffnesses around $0.5 \mathrm{~N} / \mathrm{mm}$. The stiffness bounds as a function of the out-of-plain angle can be used to calculate the boundaries of the workspace by solving the forward position kinematics for configurations where $20^{\circ}<\alpha<60^{\circ}$. Fig. (5) shows the workspace of the stage. It can be seen that it contains a cube of side $4 \mathrm{~mm}$, produced by actuator displacements ranges of about $7 \mathrm{~mm}$.

\section{Assembly}

After lamination of the frame and the mechanism structure, the resulting laminates are manually assembled with components for actuation and motion detection. To convert the rotational motion of the miniature steeper motors into input linear motion at the mechanism, the Delta LMMT uses custom-made threaten adapterconnecting the screws of each motor to a pair of linear metallic guides by means of brass bushings. The adapters have mounting holes for the subsequent attachment of the mechanism structure. The linear guides are attached to the fixed frame by 3D-printed components that offer rigidity and alignment. Attaching the mechanism structure to the adapters forces the structure

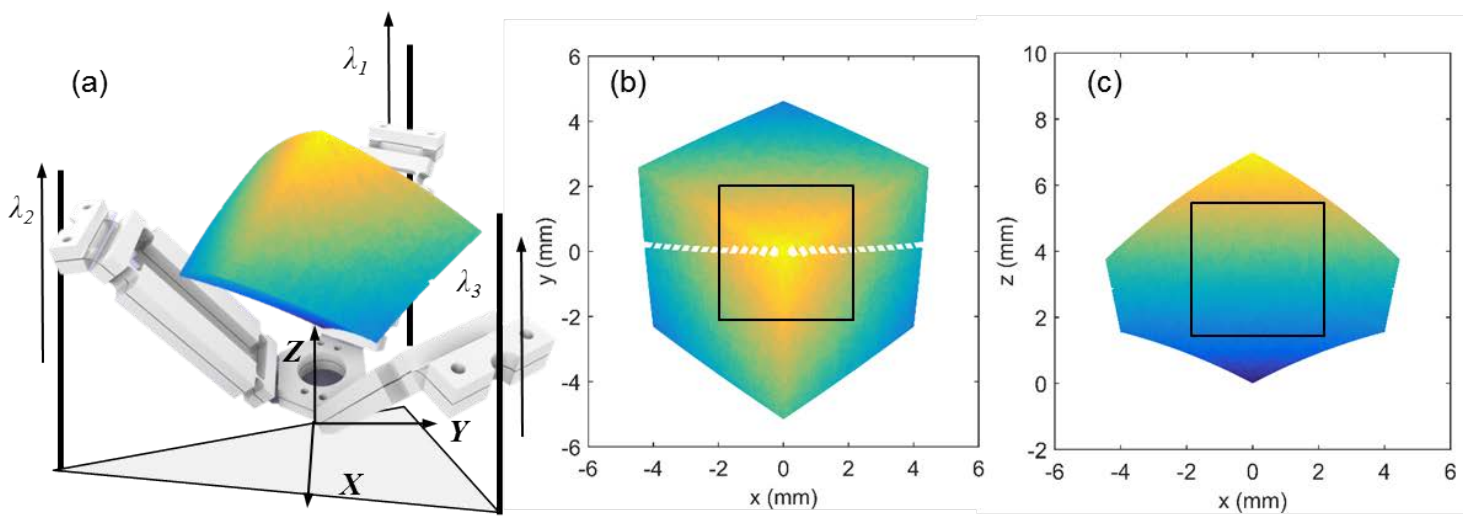

Figure 5. (a) Workspace of the end-effector of the Delta robot and orthogonal projection on (b) XY and (c) XZ planes. 

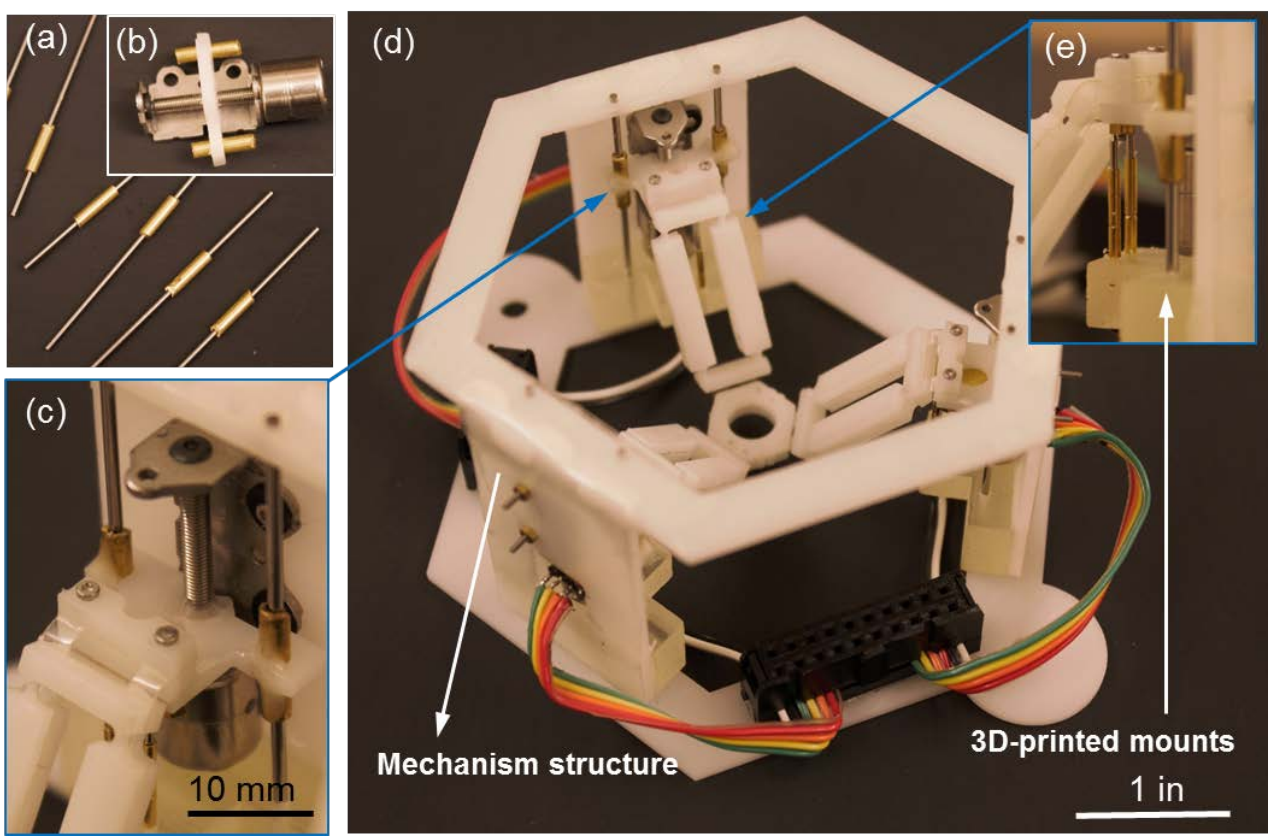

Figure. 6. Integration of components and assembly of LMMT. Components include: (a) metallic rods and brass bushings for linear bearings, (b) miniature stepper motors with plastic custom-made threaten adapter, (c) miniature screws and nuts for the attachment of the mechanism structure with the actuators, (e) pogo pins and contact electrodes for integrated limit switches and 3D-printed mounts for linear bearings and pin connectors. (d) Picture of completed Delta robot.

out of the plain creating the pop-up folding (See Fig. 6). The offset in the location of the attachment points between the mechanism structure and the frame determines the initial pop-up angle (in our case 45 ). Limit switches, created with pogo pins and copper electrodes prevent the adapters from reaching the limits of the screws.

\section{Testing}

TheDelta LMMT is driven in open loop. To yield an accurate kinematics model of the Delta mechanics and to assess the dimensional accuracy of the micromachining process, the length $l$ of the four-bar parallelograms was measured to be $28.13 \mathrm{~mm}$ using optical microscopy. The solution of the inverse kinematics problem is implemented in the computer using the open source software environment Processingto map the prescribed motion of the end-effector to the corresponding motion of the actuators. The calculated motion of the actuators is converted in number of steps and sent in form of machine code to an Arduino board in charge of interpolating the motion. The micro-controller generates the motion pulses and sends them to the drivers, which, in turn, output the corresponding voltage signals to the stepper motors.

Because of the vertical arrangement of the actuators and the symmetry of the structure, the out-ofplain kinematics are trivial in the sense that equal displacements at the actuators result in vertical motions at the end-effector of the same magnitude. To validate the in-plain kinematics, the endeffector was sequentially commanded to move to equally space points in a circumference of $4 \mathrm{~mm}$ diameter at a height of $3.5 \mathrm{~mm}$ in its workspace. A digital image correlation algorithm [18] is used to compute the displacement relative to a reference frame of the end-effector at the center of the circle. 


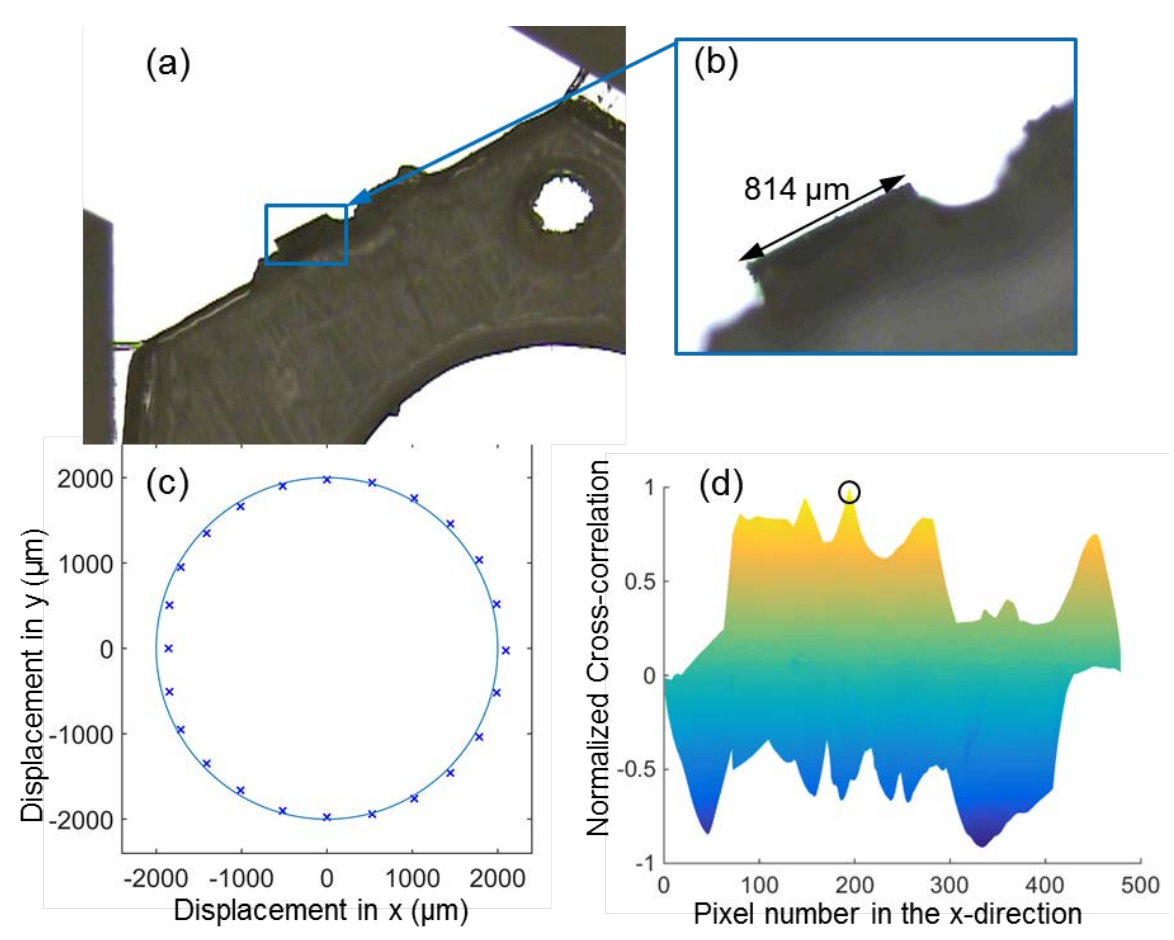

Figure. 7. (a) Reference image of end-effector, (b) selected kernel, (c) reference (in markers) and commanded circular trajectory and (d) cross-correlation algorithm along the horizontal direction of (a).

Figure 7(a) shows a frame of the end-effector at a particular position. The displacements are calculated based on the location of the kernel, in fig. 7(b), at each of the frames. Figure 7(c) shows the difference show the reference circle and the observed locations of the end-effector. The maximum error in $90 \mu \mathrm{m}$, this is expected as a result of manufacturing inaccuracies, the precision in the miniature stepper motors, differences between the model parameter and the actual length of the robot and truncation errors in the calculations. Figure 7(d) shows the displacement calculationin the $\mathrm{X}$ direction (around 200 pixels) of the cross-correlation algorithm at one of the frames. The $\mathrm{X}$ and $\mathrm{Y}$ displacements of a frame are calculated as those $\mathrm{X}$ and $\mathrm{Y}$ translations that maximize the pixel-to-pixel correlation in the chosen kernel with reference frame. This technique is limited by the pixel-to-length resolution corresponding to $12 \mu \mathrm{m}$ in our setup.

\section{Conclusions}

This paper presents the design and fabrication of a miniature Delta robot as example of this manufacturing technique to produce mesoscale electromechanical systems via lamination. The structure of the robot is adapted to be flexure-based where the flexures are design to take advantage of the lamination schemes. This example presents solutions to the integration of common components in mechanical systems such as limit switches, linear guides and actuation elements. The simplicity of the manufacturing techniquesin this process makes it easy to exploit the broad spectrum of open source hardware and software tools available for the construction and communication with the devices. These systems can find applicationsin the fields of medical industry, soft robotics, flexible electronics, machine design-iteration and elimination of errors. 
With the help of the presented stiffness analysis, we were able to characterize the overall stiffness of the mechanism. This was use to arrive to an optimal pop-up angle and estimate the size of the workspace, given the characteristics of the chosen drives.After the mechanism assembly, we demonstrated its functionality with the implementation on an open-loop controller. The precision of these devices could benefit from the implementation of closed-loop control algorithms. Ongoing research continues with the implementation of sheet metal manufacturing techniques for better accuracy, durability and holding force capabilities. We are also investigating manufacturing advances that allow the integration of distributing sensing with the lamination processes.

\section{Acknowledgements}

This work was supported in part by the National Science Foundation under grant \#1301336. Funds from theTungchao Julia Lu Professorship in the Department of MechanicalScience and Engineering also made this work possible.

\section{References}

[ P. S. Sreetharan, J. P. Whitney, M. D. Strauss and R. J. Wood, "Monolithic fabrication of 1] milimeter-scale machines," J. Micromech. Microeng., no. 22, 2012.

[ J. Dong and P. M. Ferreira, "A novel parallel-kinematics mechanisms for integrated, multi-

2] axis nanopositioning Part 1. Kinematics and design for fabrication," Precision Engineering 32 (2008) 7-19, p. 32 7-19, 2008.

[ T. Laliberte, C. M. Gosselin and G. Cote, "Practical prototyping.," Robotics \& Automation 3] Magazine, IEEE,, vol. 8(3), p. 43-52, 2001..

[ J. Correa and P. M. Ferreira, "Analysis and design for Rapid Prototyping Mechanism Using

4] Hybrid Flexural Pivots," in IEEE International COnference on Robotics and Automation, Charlotte, NC, 2015.

[ K. Alok and D. W. Rosen, "Building around inserts: methods for fabricating complex devices

5] in stereolithography.," Rapid Prototyping Journa, vol. 7(5), p. 253-262, $2001 .$.

[ C. Yonghua and Z. Chen, "Joint analysis in rapid fabrication of non-assembly mechanism.,"

6] Rapid Prototyping Journal, vol. 17(6), p. 408-417, $2011 .$.

[ M. Constantinos, J. D. Kathryn, W. Jey and A. Munshi, "Fabrication of nonassembly

7] mechanisms and robotic systems using rapid prototyping," Joural of Mechanical Design, vol. 123, p. 516, 2001.

[ E. Edqvist, N. Snis, R. C. Mohr and S. Olver, "Evaluation of building technology for mass

8] producible milimetre-sized robots using flexible printed circuit board," j. Micromech. Microeng., vol. 19, 2019.

[ J. P. Whitney, P. S. Streetharan, K. Y. Ma and R. J. Wood, "Pop-up book MEMS," j. 9] Micromech. Microeng, vol. 21, 2011.

[ D. M. Aukes, O. Ozcan and W. Robert J., "Monolithic Design and Fabrication of a 2-DOF 10] Bio-Inspired Leg Transmition," in Biomimetic and Biohybrid Systems, Milan, Italy, 2014. 
[ N. Ahmed, C. Dagdviren, J. Rogers and P. M. Ferreira, "Active Polymeric Composite 11] Membranes for Localized Actuation and Sensing in Microtransfer Printing," JOURNAL OF MICROELECTROMECHANICAL SYSTEMS,, 2015.

[ A. M. Hoover and R. S. Fearing, "Fast sclae prototyping for folded millirobots," Pasadena, 12] CA, 2008.

[ R. Clavel, Conception d'un robot parallèle rapide à 4 degrés de liberté, Ph.D. Thesis, EPFL, 13] Lausanne, Switzerland, 1991.

[ R. Williams, "The Delta Parallel Robot: Position Kinematics Solutions," 2014. [Online]. 14] Available: www.ohio.edu/people/williar4/html/pdf/DeltaKin.pdf,.

[ N. Lobontiu, Compliant mechanism: design of flexures hinges, CRC press, 2003.

15]

[ L. Howel, Compliant Mechanisms, New York: Wiley, 2001.

16]

[ J.Dong and P. M. Ferreira, "A novel parallel-kinematics mechanism for integrated, multi-axis 17] nanopositioning Part 2: Dynamics, control and performance analysis.," Precision Engineering 32 (2008) 20-33, p. 32 20-33, 2008.

[ Mathworks, "Registering an Image Using Normalized Cross-Correlation- MATLAB and 18] Simulink $\quad 2015 . \quad$ Example," [Online]. Available: http://www.mathworks.com/help/images/examples/registering-an-image-using-normalized-crosscorrelation.html. 